\title{
Mar de fondo: Propuesta para mitigar inundaciones en la calle Juan Serrano de la ciudad de Acapulco, Guerrero, México
}

\author{
Ground swell: Proposal to mitigate flooding on Juan Serrano Street \\ in Acapulco city, Guerrero, Mexico
}

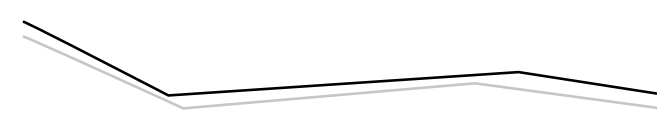

Cynthia I. Jaimes Tenorio*凶, Esteban R. Guinto Herrera*, Roberto
Arroyo Matus*

Jaimes Tenorio, C. I., Guinto Herrera, E. R., \& Arroyo Matus, R. (2017). Mar de fondo: Propuesta para mitigar inundaciones en la calle Juan Serrano de la ciudad de Acapulco, Guerrero, México. Investigación y Ciencia de la Universidad Autónoma de Aguascalientes, 25(72), 75-81.

RESUMEN

Se comenta el problema de inundación de la calle Juan Serrano y parte de la Av. Costera Miguel Alemán de la ciudad de Acapulco, Guerrero, causado por el fenómeno mar de fondo. Se explica cómo se ha presentado el fenómeno antes referido, así como los efectos y la alteración de la circulación del tránsito vehicular sobre la Av. Costera Miguel Alemán. A partir de un análisis cualitativo de la problemática y topografía del sitio, se presenta una propuesta para mitigar las inundaciones de la zona antes mencionada, causadas por el fenómeno referido.

\section{ABSTRACT}

The flooding problem on Juan Serrano Street and part of Costera Miguel Alemán Avenue in Acapulco city, caused by ground swell phenomenon, is discussed. The way in which the phenomenon has occurred, as well as the effects and the alteration of the movement of traffic in Costera Miguel Alemán Avenue, are explained. A proposal to mitigate the flooding of the above mentioned area, caused by

Palabras clave: inundación; mar de fondo; mitigación; ciudad de Acapulco.

Keywords: flooding; ground swell; mitigation; Acapulco city.

Recibido: 15 de noviembre de 2016, aceptado: 18 de septiembre de 2017

* Facultad de Ingeniería, Universidad Autónoma de Guerrero. Av. Lázaro Cárdenas s/n, Col. La Haciendita, C. P. 39090, Chilpancingo, Guerrero, México. Correo electrónico: c.jaimes@hotmail.com; rguinto2002@

$凶$ yahoo.com.mx; arroyomatus@hotmail.com

Autor para correspondencia the ground swell phenomenon, is presented. The proposal is based on a qualitative analysis of the actual situation of the problem and topography.

\section{INTRODUCCIÓN}

En los últimos años, en la ciudad de Acapulco se han observado inundaciones en diferentes avenidas de la ciudad, por lluvias con precipitaciones a partir de los 100 mm, según la Comisión Nacional del Agua (CNA, 2015). La calle Juan Serrano y una porción de la Av. Costera Miguel Alemán a menudo son inundadas bajo estas lluvias (comunicación personal con el coordinador de Protección Civil y Bomberos de Acapulco de Juárez, Guerrero) y en presencia del fenómeno mar de fondo, definido como el movimiento de las olas que se propaga fuera de la zona donde se ha generado, pudiendo llegar a lugares muy alejados (Mandujano, 2006). Las acciones llevadas a cabo por autoridades, como el uso de retroexcavadoras para abrir un canal sobre la playa y bombas de agua tipo Vactor, con el fin de regresar al mar parte del agua que salió del mismo, también el retiro de arena, basura y automóviles acarreados por las olas o por corrientes de aguas pluviales, y el desazolve de alcantarillas, han sido encaminadas a solucionar el problema, pero no a las causas.

La zona en estudio se ubica en las calles antes mencionadas, entre el hotel Playa Suites y el hotel Emporio. Esta zona, desde el año 2008, de manera marcada viene exhibiendo el fenómeno tratado, el cual se presenta en varias ocasiones durante la temporada de lluvias que inicia en el mes de mayo y termina en el mes de noviembre de cada año. El fenómeno genera un incremento del nivel del mar 


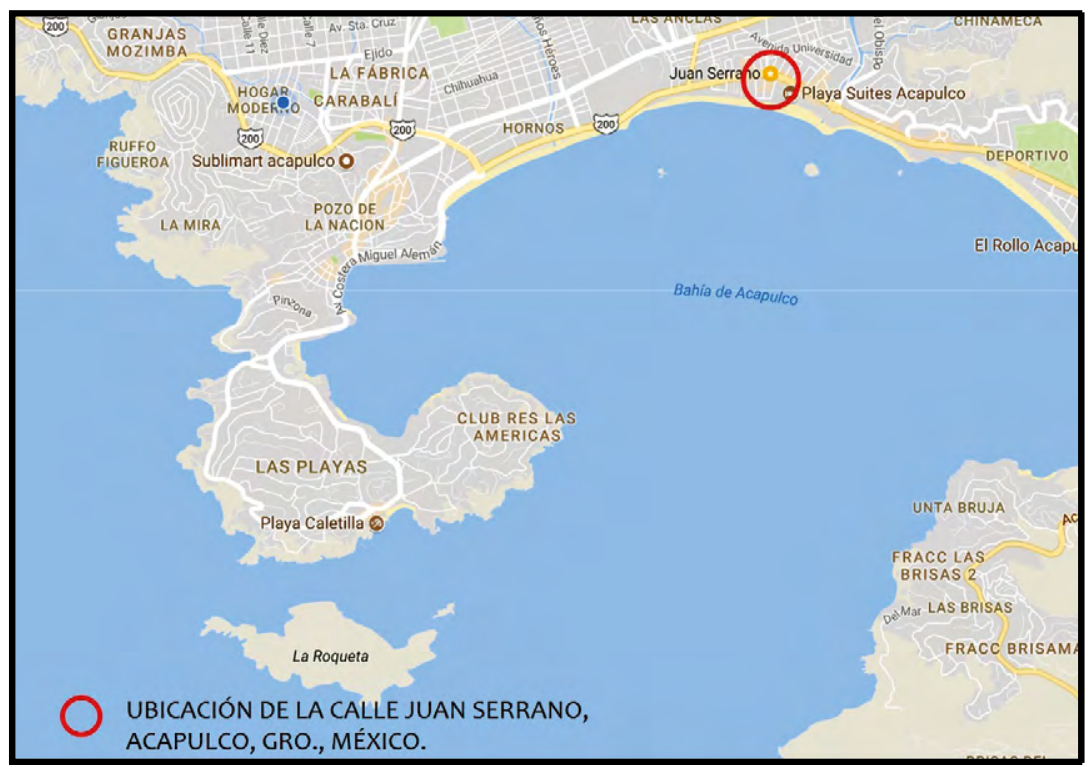

Figura 1. Ubicación de la zona en estudio.

Imagen modificada de Google Maps 2015.

provocando la entrada de agua en las calles ya citadas. Al momento del estudio se pudo observar que los niveles de desplante de los hoteles Playa Suites y Emporio están $2.00 \mathrm{~m}$ por arriba del nivel que alcanza el mar, lo que ayuda a que no se inunden.

En este trabajo se presenta una propuesta para mitigar las inundaciones en la zona de estudio, causadas por el fenómeno mar de fondo. Cabe mencionar que hasta antes del estudio no se tiene registro alguno de soluciones o propuestas de solución al problema similares a la que aquí se presenta.

\section{Antecedentes}

El área urbana de la ciudad de Acapulco, Guerrero, ha tenido un crecimiento caótico (Carrascal \& Pérez Villegas, 1998); sin embargo, la fisonomía original de la bahía de Acapulco e infraestructura turística, a lo largo de su historia ha sido modificada para dar una buena imagen y atender a los turistas (SECTUR, ANIDE, CESTUR, \& Conacyt, 2013), la construcción de la zona hotelera del lado de la franja de playa es un caso de ello; aunado a esta alteración, se ha presentado un incremento en el número de habitantes del municipio de Acapulco (INEGI, 2010), originando con esto más deshechos, como basura y -en ocasiones- escombros, que en tiempos de lluvia son arrastrados a la bahía.

Debido al cambio climático en la Tierra, los fenómenos meteorológicos se presentan con mayor severidad (Coumou \& Rahmstorf, 2012). Los efectos del fenómeno mar de fondo generan problemas para la circulación de los ciudadanos, poniendo en peligro su integridad física. Del mismo modo afecta al tránsito vehicular sobre la Av. Costera Miguel Alemán y a los autos que estacionan en la calle Juan Serrano. Cotidianamente esta calle se usa como estacionamiento público; sin embargo, cuando el fenómeno estudiado se presenta, las marejadas se incrementan y el nivel del agua de mar sube, inundando las calles ya referidas. Las inundaciones en esa zona también ocurren cuando se presentan lluvias moderadas o fuertes. Los vehículos estacionados en la calle son cubiertos por el agua y/o arrastrados hacia el mar, causando con ello pérdidas económicas considerables a sus propietarios.

En la figura 1 se muestra la bahía de Acapulco; el círculo rojo indica la ubicación de la zona en estudio. Las figuras 2 y 3 muestran algunos de los efectos y escenarios que se presentan durante las inundaciones ocasionadas por el mar de fondo en las calles referidas con antelación. En la figura 2 (a) puede observarse una inundación de la calle Juan Serrano debido al fenómeno varias veces mencionado; se observan autobuses y un automóvil atrapados, los cuales se encontraban estacionados sobre esta vialidad antes del evento. En la figura 2 (b) se observa otra inundación de la misma calle, donde el mar salió a unos $200 \mathrm{~m}$ desde la pleamar; al fondo de la imagen puede verse parte de la bahía de Acapulco. En la figura 3 se muestra una inundación en la Av. Costera Miguel Alemán; en ella pueden 


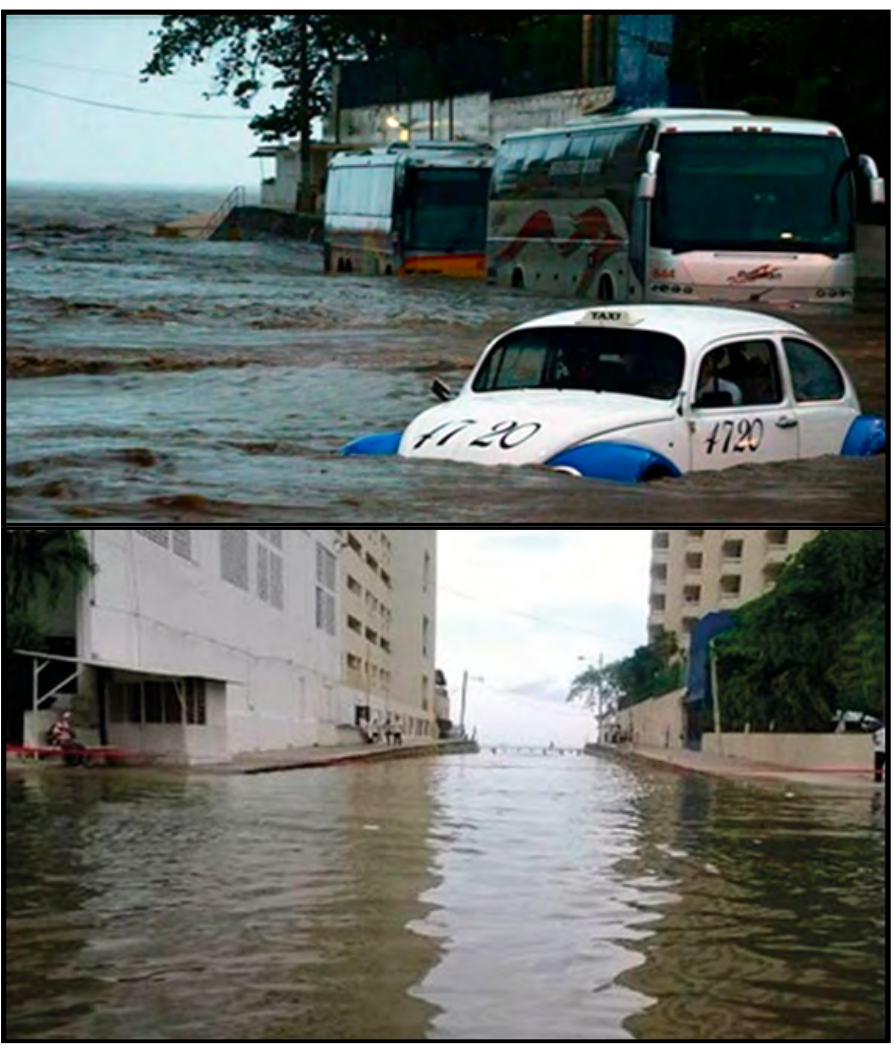

Figura 2. Inundación de la calle Juan Serrano por el fenómeno mar de fondo.

a) Fotografía de Sánchez (2014), b) Fotografía de Trujillo (2014).

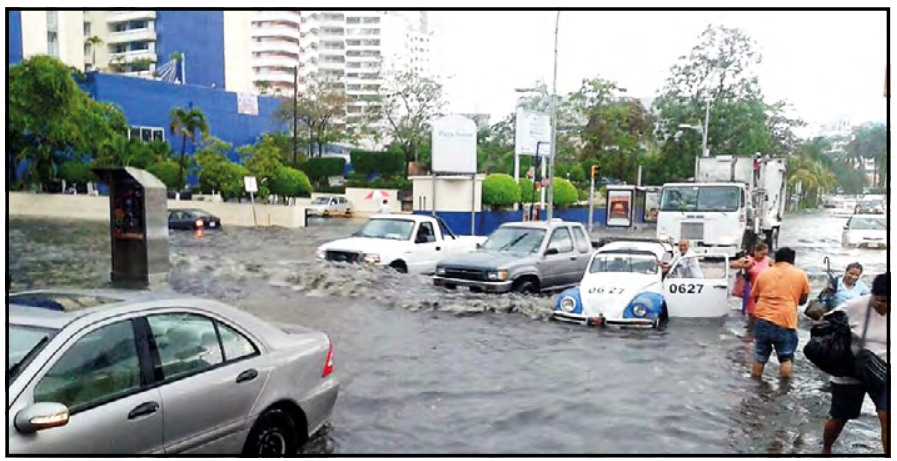

Figura 3. Inundación de Av. Costera Miguel Alemán por lluvias. Fotografía de staff de redacción periódico Libertad (2013).

observarse dificultades para el tránsito vehicular y peatonal.

Cada año el fenómeno hace que el oleaje del mar arroje arena, incrementando su cota en $1.87 \mathrm{~m}$ y haciendo que el agua de mar se desborde hacia las calles de la zona en estudio, a una altura máxima de $1.00 \mathrm{~m}$ sobre el nivel de la Av. Costera Miguel Alemán (figura 4).
Durante el huracán Paulina, en 1997, el indicador de inundación por marea de tormenta (IIMT) en la ciudad de Acapulco, determinado a partir de la máxima amplitud de marea de tormenta registrada, fue de $5.24 \mathrm{~m}$ (Zepeda Ramos \& Sánchez Pérez, 2001). Las áreas más vulnerables a inundaciones por marea de tormenta son la playa Icacos, la desembocadura del río Costa Azul y la zona de la playa Papagayo, frente a las desembocaduras del río Camarón y Grande, sitios donde se tiene una topografía muy baja (SECTUR et al., 2013).

La bahía de Acapulco, debido a su geomorfología (cerrada con puntas que protegen sus flancos del canal de acceso por el sur), únicamente acepta oleaje del sector sur; estos oleajes entran de manera franca a la bahía, pero sufren refracción, lo que genera disminución de su energía (SECTUR et al., 2013). La ciudad de Acapulco tiene una topografía tipo anfiteatro, con diferentes cuencas alrededor que permiten bajar las aguas pluviales provenientes de los cerros para conducirlas al mar; en su paso por la ciudad se conectan con las rejillas pluviales de acero, que permiten el desagüe de la parte baja para evitar inundaciones y cumplir con el ciclo de estos residuos. La ciudad tiene un riesgo alto de inundación en la zona costera y un riesgo medio en la zona alejada de la costa. La Dirección de Conservación y Mantenimiento es una dependencia del gobierno municipal encargada del mantenimiento y desazolve de las rejillas, tiene un programa que existe hace más de 20 años y es financiado por el gobierno federal. Cada año se ejercen recursos económicos para la ejecución de estos trabajos (comunicación personal con el jefe de operatividad de la Dirección de Conservación y Mantenimiento de Acapulco de Juárez, Guerrero); sin embargo, el deterioro de las rejillas es constante por la salinidad existente en la brisa natural, que con esfuerzos y uso de antioxidantes permiten que el acero llegue al término de su vida útil; también debe considerarse el robo de las tapas (comunicación personal con Dirección de Conservación y Mantenimiento, H. Ayuntamiento de Acapulco, Guerrero, 2015). En la dependencia a cargo de estas rejillas pluviales no cuenta con registro de las tapas existentes, se realizó una inspección ocular por parte del autor de este artículo y se verificó que en la calle Juan Serrano no existen físicamente rejillas pluviales.

Hipótesis: Un muro de contención, en la calle Juan Serrano, permitirá mitigar las inundaciones que se generan por el fenómeno mar de fondo en esa zona. 


\section{MATERIALES Y MÉTODOS}

Se llevó a cabo un análisis cualitativo de la problemática y topografía del sitio, se observó que las aceras de los hoteles Playa Suites y Emporio tienen una cota más elevada a la calle Juan Serrano. Se consultó el historial de eventos y se llevó a cabo un levantamiento topográfico, antes y después de presentarse el fenómeno, con el fin de observar la diferencia en las cotas de los niveles de arena. Uno de los perfiles topográficos puede verse en la figura 4 .

Propuesta de solución:

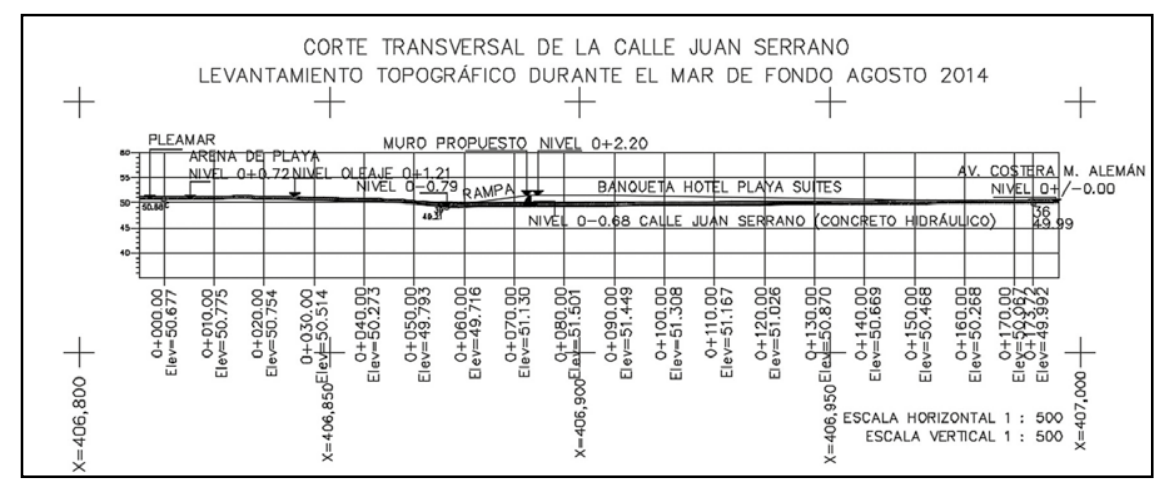

Figura 4. Perfil topográfico durante el mar de fondo.

Elaboración propia.

Con base en la información recabada y en los tipos y funciones de estructuras de protección costera (Salles Afonso de Almeida \& Silva Cazarín, 2004), se plantea como propuesta de solución un muro de mampostería de piedra con dentellón, que se ubicará en el punto más alto de las banquetas de los hoteles Emporio y Playa Suites, atravesando la calle Juan Serrano, para impedir el paso del agua del mar hacia la calle (figuras 5 y 6 ).

El muro propuesto tendrá rejillas de desagüe que canalizarán el agua pluvial que se estanque en la calle Juan Serrano y la conducirán a un foso de absorción que estará ubicado dentro de la arena de la playa. Tales rejillas tendrán una trampa para evitar que desechos de basura tapen el conducto, sin embargo, tendrán que desazolvarse periódicamente de manera preventiva.

Para evitar que el muro de contención sea un obstáculo visual, en la corona del muro se propone un cantiliver, que servirá de mirador para que los turistas y peatones en general transiten por la calle antes mencionada.

Para el cálculo de la base del muro de piedra braza (B) se usa la ecuación 1.

$$
B=H \sqrt{\frac{\gamma}{\gamma^{\prime}}}
$$

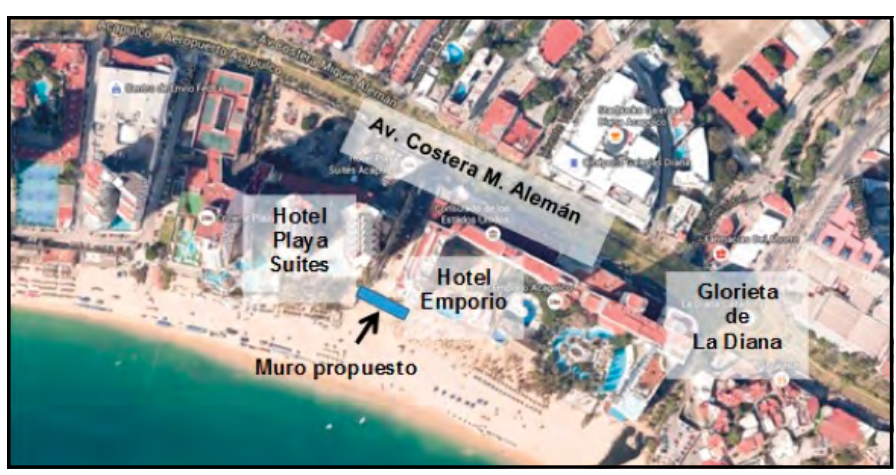

Figura 5. Ubicación del muro propuesto.

Imagen modificada de Google Earth 2015.

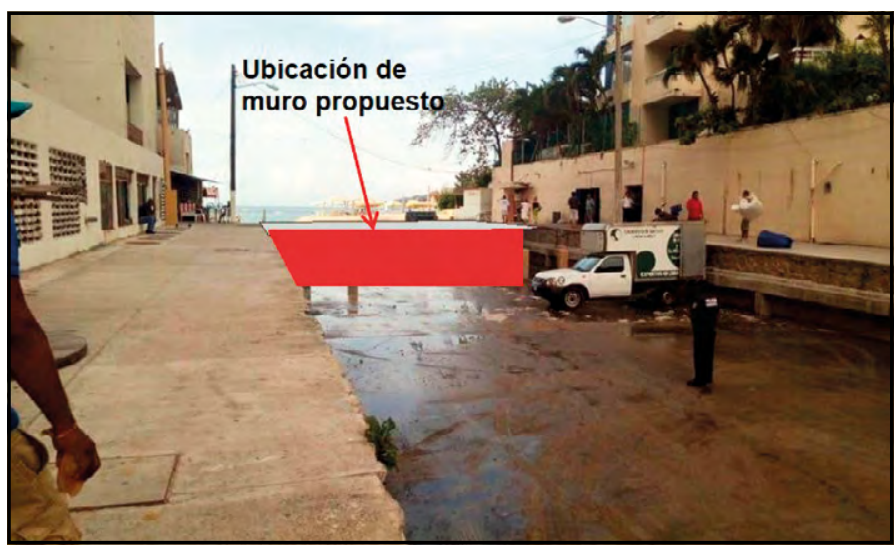

Figura 6. Montaje en fotografía de la ubicación del muro de piedra. Fotografía tomada por los autores. 


\section{IIVESTIGAGIÓn Y CUERCIA DE LA UחIVERSIDAD AUTÓNOMA

Donde:

$B=$ Base del muro de contención de piedra braza

$\mathrm{H}=$ Altura propuesta del muro de contención

$\gamma^{\prime}=$ Peso específico del agua de mar

$\gamma=$ Peso específico de la arena de playa mojada

Para el cálculo de la corona del muro de contención (B') se usa la ecuación 2.

$$
B^{\prime}=h \sqrt{\frac{\gamma}{\gamma^{\prime}}}
$$

Donde:

$\mathrm{B}^{\prime}$ = Corona del muro de contención de piedra braza

$\mathrm{H}=$ Nivel del oleaje

$\gamma^{\prime}=$ Peso específico del agua de mar

$\gamma=$ Peso específico de la arena de playa mojada

Según el Manual de muros de contención de la SAGARPA (Llanderal Cázares \& Fernández Reynoso, 2009), las siguientes medidas de dentellón son aplicables a muros con altura de $2.00 \mathrm{~m}$ a $6.00 \mathrm{~m}$ (figura 7):

$\mathrm{b} 2=0.80 \mathrm{~m}$

$\mathrm{bl}=0.30 \mathrm{~m}$

$\mathrm{h}=0.60 \mathrm{~m}$

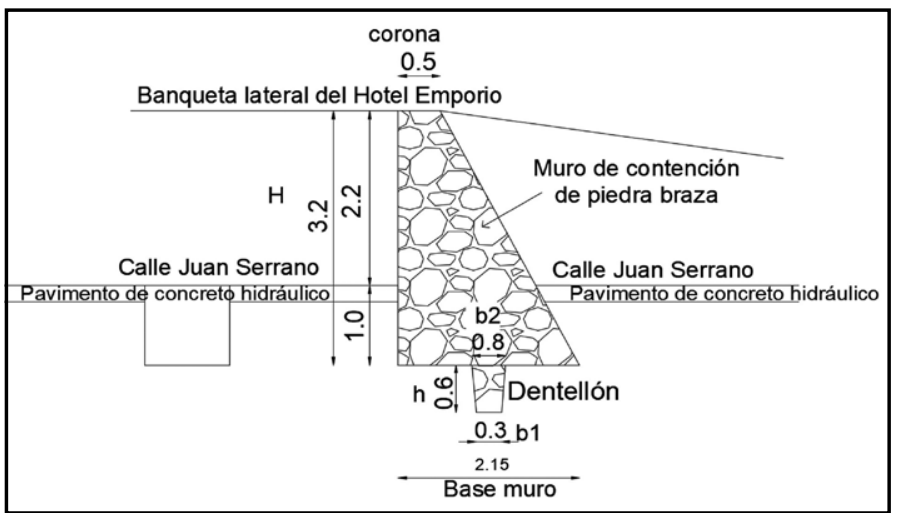

Figura 7. Muro de contención.

Elaboración propia.

Para el cálculo de la velocidad del agua pluvial en el canal se utilizan las fórmulas de Chézy y Manning, ecuaciones 3 y 4.

$$
\begin{gathered}
V=C \sqrt{R * S} \\
V=\frac{1}{n} R^{2 / 3} \sqrt{S}
\end{gathered}
$$

Donde:

$V=$ Velocidad del agua pluvial

$\mathrm{R}=$ Radio hidráulico (sección/parámetro mojado)

$\mathrm{n}=$ Parámetro de rugosidad

$\mathrm{C}=$ Coeficiente de Manning

$S=$ pendiente del canal

Para determinar el escurrimiento superficial en estructuras hidráulicas menores como las utilizadas en fraccionamientos, en donde no hay almacenamiento ni retención de agua pluvial, se utiliza el método racional (Beltrán, 2011) que está definido por la ecuación 5 .

$$
Q=C * i d * A * 0.27777
$$

\section{Donde:}

$Q=$ Gasto del escurrimiento superficial en $\mathrm{m}^{3} / \mathrm{s}$

$C=$ Coeficiente de escurrimiento ponderado para el área tributaria por analizar, es igual al porcentaje de la lluvia que aparece como escurrimiento directo

$\mathrm{Id}=$ Intensidad media de la lluvia en $\mathrm{mm} / \mathrm{h}$ para una duración igual al tiempo de concentración de la cuenca

$\mathrm{A}=$ Área tributaria del drenaje por analizar en $\mathrm{km}^{2}$

Para el cálculo del área, se usa la ecuación 6.

$$
A=\frac{Q}{V}
$$

Donde:

$Q=$ Gasto del escurrimiento superficial en $\mathrm{m}^{3} / \mathrm{s}$

$V=$ Velocidad del agua pluvial

$\mathrm{A}=$ área total de tubería requerida

Para el cálculo del diámetro de la tubería se usa la ecuación 8, que se obtiene de la ecuación 7.

$$
\begin{gathered}
A=\left(\frac{1}{4}\right) \pi D^{2} \\
D=\sqrt{\frac{4 A}{\pi}}
\end{gathered}
$$

Donde:

$\mathrm{D}=$ Diámetro del tubo

$\mathrm{A}=$ Área de tubería requerida

\section{Criterio estructural de la losa de terraza}

Se propone una terraza que funcionará como 
mirador y que unirá las banquetas de los hoteles Playa Suites y Emporio; la losa quedará apoyada sobre el muro de contención con un ancho de 3.00 $\mathrm{m}$ y de largo $12.55 \mathrm{~m}$, siendo este el mismo ancho de la calle Juan Serrano. La losa estará empotrada a la corona del muro, con el armado a $0.60 \mathrm{~m}$ de profundidad; estará soportada por trabes cada 2.00 $m$ y una cadena principal del mismo ancho de la corona del muro (figura 8).

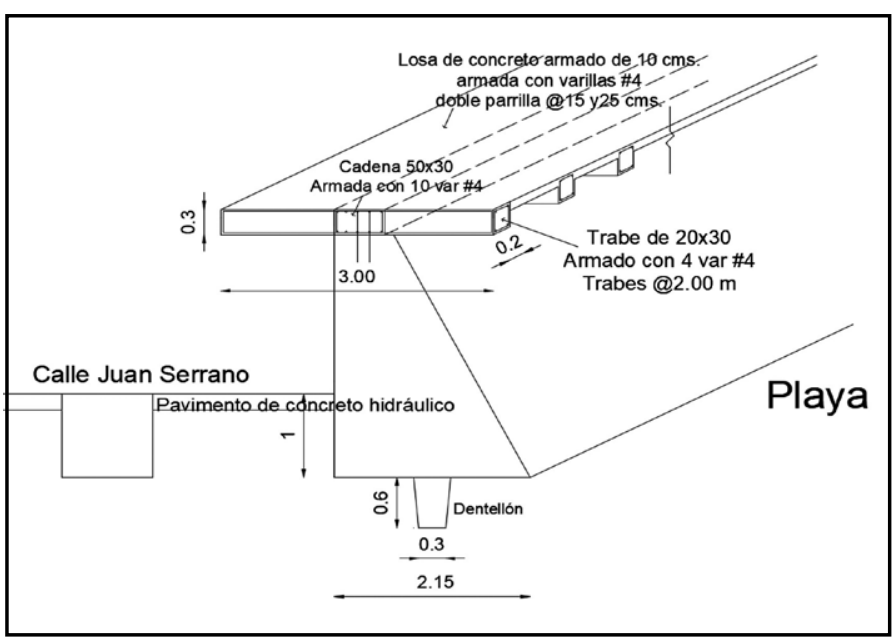

Figura 8. Estructura de la terraza.

Elaboración propia.

RESULTADOS

Se realizaron los cálculos necesarios para el muro de contención, la losa de terraza y la tubería para el desagüe de aguas pluviales, con el fin de mitigar las inundaciones de la zona en estudio, causadas por el fenómeno investigado en el presente trabajo. A continuación se presentan los resultados.

La distancia de los tubos al foso de absorción que se ubicará en la playa tendrá una separación de $20.00 \mathrm{~m}$, a partir de donde termina la cimentación del muro de contención (figuras 9 y 10).

\section{Propuesta final}

El diseño final consiste en un muro de piedra braza sobre el cual descansará un cantiliver de $3.00 \mathrm{~m}$, que trabajará como terraza (figura 11). El muro de contención detendrá el paso del oleaje provocado por el mar de fondo. Para el desagüe de las aguas pluviales que se concentran en la calle Juan Serrano se propone una rejilla pluvial junto al muro, que conducirá el agua por gravedad a un pozo de absorción que se ubicará bajo la arena de la playa (figuras 9 y 10).

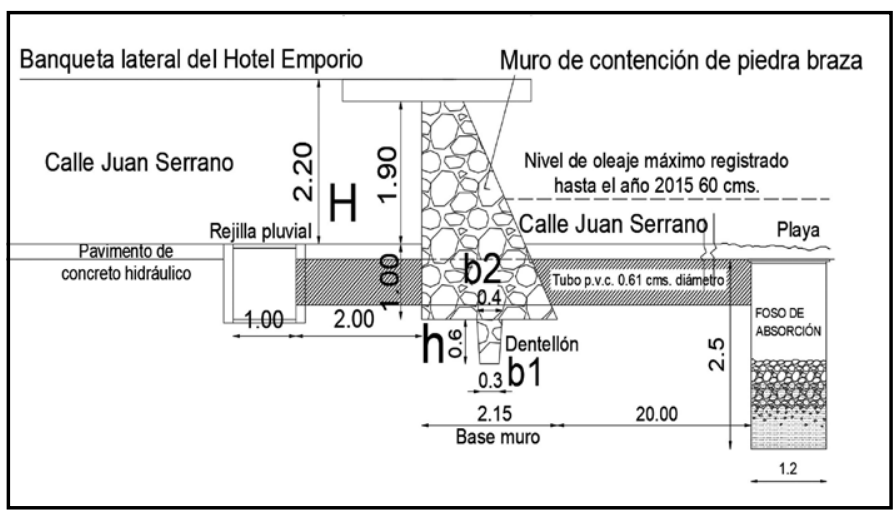

Figura 9. Alzado del muro de contención.

Elaboración propia.

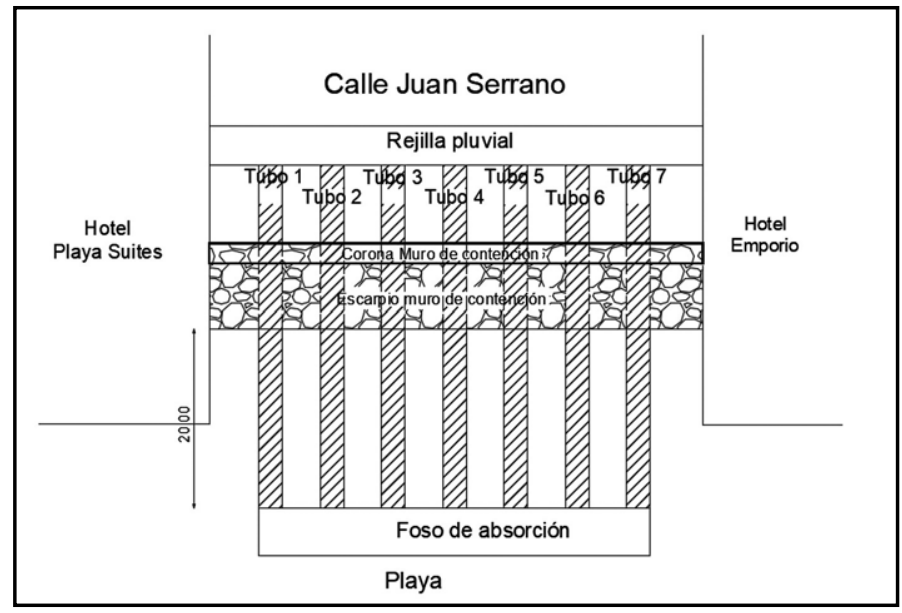

Figura 10. Planta de tubería.

Elaboración propia.

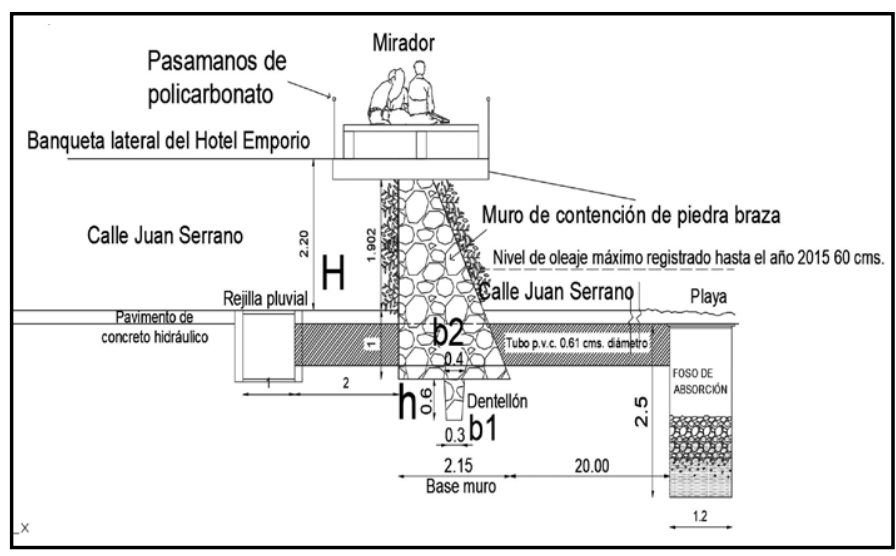

Figura 11. Propuesta final del muro de contención. Elaboración propia. 
DISCUSIÓN

Los resultados obtenidos hacen que en la actualidad se cuente con una propuesta de solución a la inundación de la zona en estudio, causado por el fenómeno mar de fondo.

Es de suponer que los efectos que tendrá el bloqueo de la salida de la marea provocada por el fenómeno estudiado serán mínimos, ya que el agua que ya no saldrá por la calle Juan Serrano se distribuirá por la playa.

\section{CONCLUSIONES}

El muro de contención propuesto está diseñado para recibir los impactos provocados por el oleaje del mar de manera preventiva; es una propuesta de solución que permitirá detener el flujo del agua y, mediante un foso de absorción, ayudará a desalojar el líquido de origen pluvial retenido en la calle Juan Serrano. Una vez construido, el muro de contención propuesto solucionará el problema de inundación de la calle Juan Serrano y parte de la Av. Costera Miguel Alemán.

Se cuenta con una propuesta de muro de contención que permitirá a las autoridades construirlo con el fin de mitigar las inundaciones que se generan por el fenómeno mar de fondo en las calles citadas.

Las perspectivas son las siguientes:

- Realizar un estudio, con el fin de saber los posibles efectos que tendrá el bloqueo de la salida de la marea provocada por el mar de fondo.

- Difundir la propuesta al Colegio de Ingenieros Civiles.

- Difundir la propuesta al gobierno municipal, con el fin de que esta obra sea ejecutada mediante financiamiento público.

REFERENCIAS

- Beltrán, E. (2011). Proyectos ejecutivos de las redes de distribución de agua potable, drenaje sanitario y drenaje pluvial del Fraccionamiento "Rancho Bellavista", municipio de Querétaro, Edo. de Querétaro (Tesis de Licenciatura en Ingeniería Civil). UNAM, México. Recuperada de http://132.248.52.100:8080/xmlui/handle/132.248.52.100/483

- Carrascal, E., \& Pérez Villegas, G. (1998). Ocupación territorial y deterioro ambiental ocasionado por la expansión urbanoturística en Acapulco, Guerrero. Investigaciones geográficas, $37,111-124$.

- Comisión Nacional del Agua. (2015). Resúmenes mensuales de temperaturas y lluvia. 2017 [Portal electrónico]. Recuperado de http://smn.conagua.gob.mx/es/climatologia/temperaturas-ylluvias/resumenes -mensuales-de-temperaturas-y-lluvias.

- Coumou, D., \& Rahmstorf, S. (2012). A decade of weather extremes. Nature climate change, 2, 491-496. doi: 10.1038/nclimatel 452

- Instituto Nacional de Estadística y Geografía. (2010). Información por entidad. Número de habitantes. 2017. Recuperado de http://cuentame.inegi.org.mx/monografias/informacion/ gro/poblacion/dinamica.aspx?tema=me\&e=12

- Llanderal Cázares, R., \& Fernández Reynoso, D. S. (2009). Muros de contención [Manual en pdf]. México. SAGARPA.

- Mandujano, L. (2006). Fenómeno Mar de Fondo. México: IPN.

- Salles Afonso de Almeida, P., \& Silva Cazarín, R. (2004). Infraestructura de protección costera. En E. Rivera-Arriaga, G. J. Villalobos-Zaoata, I. Azuz Adeath, \& F. Rosado May (Eds.), El manejo costero en México (pp. 179-190). México: Universidad de Quintana Roo-Universidad Autónoma de CampecheSEMARNAT-CETYS Universidad.
- Sánchez, L. (5 de julio de 2014). Aún continúa inundada parte de la Costera Miguel Alemán por la contracorriente marina de Mar de Fondo en Acapulco. Punto X Punto Noticias [Blog informativo]. Recuperado de http://puntoxpuntonoticias. blogspot.mx/2014/07/aun-continua-inundada-parte-de-la.html

- Secretaría de Turismo, Asociación Nacional de Investigación y Desarrollo, Centro de Estudios Superiores de Turismo, \& Consejo Nacional de Ciencia y Tecnología. (2013). Vulnerabilidad del destino turístico Acapulco. En Estudio de la vulnerabilidad y programa de adaptación ante la variabilidad climática y el cambio climático en diez destinos turísticos estratégicos, así como propuesta de un sistema de alerta temprana a eventos hidrometeorológicos extremos (Sección II, 31 pp.). Proyecto 165452. México: Academia Nacional de Investigación y Desarrollo (ANIDE).

- Staff de Redacción. (29 de mayo de 2013). Inundaciones, arrastres de basura, tierra, piedras y choques vehiculares por «Bárbara». Libertad periodismo independiente [Periódico en línea, Dir. F. Cárdenas López]. Recuperado de http://www. libertadguerrero.net/2013/05/inundaciones-arrastres-debasura-tierra.html.

- Trujillo, J. (4 de julio de 2014). Se desborda el mar en zona Dorada de Acapulco. Milenio.com. Sección Estados. Recuperado de http://www.milenio.com/estados/Zona_ Dorada_de_Acapulco-se_deborda_el_mar-fondo_de_ mar-exhortan_a_banistas_y_a_la_poblacion_a_tomar_ precaciones_0_329367433.html

- Zepeda Ramos, O., \& Sánchez Pérez, T. A. (2001). Atlas nacional de riesgos de la República Mexicana: Diagnóstico de peligros e identificación de riesgos de desastres en México. México: CENAPRED. 